\title{
Free vibration analysis of solar functionally graded plates with temperature-dependent material properties using second order shear deformation theory
}

\begin{abstract}
Second-order shear deformation theory (SSDT) is employed to analyze vibration of temperature-dependent solar functionally graded plates (SFGPô). Power law material properties and linear steady-state thermal loads are assumed to be graded along the thickness. Two different types of SFGPôs such as ZrO2/Ti-6Al-4V and Si3N4/SUS304 are considered. Uniform, linear, nonlinear, heat-flux and sinusoidal thermal conditions are imposed at the upper and lower surface for simply supported SFGPs. The energy method is applied to derive equilibrium equations, and solution is based on Fourier series that satisfy the boundary conditions (Navierôs method). Non-dimensional results are compared for temperaturedependent and temperature-independent SFGPQ̂ and validated with known results in the literature. Numerical results indicate the effect of material composition, plate geometry, and temperature fields on the vibration characteristics and mode shapes. The results obtained using the SSDT are very close to results from other shear deformation theories.
\end{abstract}

Keyword: FGM; Second-order shear deformation; Solar functionally graded plate; Temperature-dependent properties; Vibration analysis 